\title{
Osteoderm distribution has low impact on the centre of mass of stegosaurs
}

\author{
H. Mallison \\ Museum für Naturkunde - Leibniz-Institut für Evolutions- und Biodiversitätsforschung \\ an der Humboldt-Universität zu Berlin, Berlin, Germany \\ Correspondence to: H. Mallison (heinrich.mallison@mfn-berlin.de)
}

Received: 7 November 2013 - Revised: 13 February 2014 - Accepted: 14 February 2014 - Published: 7 March 2014

\begin{abstract}
It has been hypothesized that the pronounced differences of stegosaur humeral shapes, with large forms having more slender and small forms having more robust humeri, may be explained by a difference in relative centre of mass (COM) placement caused by differing distributions of osteoderms. To test this hypothesis, digital 3-D models of the bones and osteoderms of the Tanzanian stegosaur Kentrosaurus aethiopicus and of the North American stegosaur Stegosaurus armatus were used to create a 3-D computeraided design life reconstruction. On these models osteoderm placement was varied drastically, recreating both existing and hypothetical forms. These models show that COM position varies somewhat with realistic osteoderm distributions, but insufficiently to explain major differences in humeral shape. The uniform weight distribution between forelimbs and hindlimbs found between the two taxa also casts doubt on the hypothesis that differences in relative COM position caused by other factors than osteoderm distribution can explain differences in humeral robustness.
\end{abstract}

\section{Introduction}

Maidment et al. (2012) hypothesized that osteoderm placement may have had a significant influence on centre of mass (COM) position, enough so to explain the proportional differences in thyreophoran humeri they found. They found that "the largest stegosaurs have relatively slender humeri in comparison with the smallest members of the clade" (Maidment et al., 2012), and proceed as follows (here treated as Hypothesis 1): "This could be due to changes in the center of mass related to distribution of dermal armor; for example, Kentrosaurus, the smallest stegosaur in the sample, is known to have possessed parascapular spines [Galton (1982)]. Despite the large number of individuals of Stegosaurus known, no parascapular spine belonging to the genus has ever been discovered [Galton and Upchurch (2004)]. The additional mass of these large dermal spines in the shoulder region might have caused the center of mass to be located further anteriorly in Kentrosaurus than in Stegosaurus" (Maidment et al., 2012).

In this context it is important to note that Hennig (1925) and Janensch (1925) as well as Galton (1982) listed the osteoderms in question as parasacral spikes, not parascapular. Mallison (2011c) agreed with this assessment. The bases of all osteoderms show a rugose texture that apparently relates to attachment of tough connective tissues, and a similar mark of identical size to the base of the spikes in question can be found on the dorsal surface of the pelvic shield in all Kentrosaurus hips. Additionally, there is a good match in shape between spike and hip. Disagreement with a placement on the hip is based on the presence of somewhat similarly shaped scapular spikes in Gigantspinosaurus, which were found in articulation with the scapulae, proving beyond any doubt that at least one species of stegosaur had parascapular points (Ouyang, 1992). However, the spike shape seems to differ quite significantly, based on evaluation of photographs provided by S. Hayashi. In any case, Hypothesis 1 of Maidment et al. (2012) is worth exploring in detail, and is here tested using CAD methods.

Maidment et al. (2012) suggest a further cause of the apparent overall robustness of stegosaur humeri in general: "The robustness of thyreophoran and particularly stegosaur humeri could be interpreted to indicate that the center of mass of stegosaurs was located more anteriorly than in other 
ornithischians, so that the humerus was required to support proportionally more of the body mass."

Maidment et al. (2012), however, point out that "Henderson [(1999)] modelled the center of mass of several dinosaurs and showed that the center of mass in Stegosaurus was located as far posteriorly as it was in bipedal taxa such as Tyrannosaurus." They then proceed to suggest an alternative suggestion, here treated as Hypothesis 2: "Alternatively, the robustness of stegosaur humeri could be related to a specific behaviour. For example, it has been suggested that stegosaurs utilized a tripodal stance [...]; perhaps increased stress on the humerus was generated during rearing as a result of pushing off from the ground."

This hypothesis will also be discussed below.

Abbreviations: CAD - computer-aided design; COM centre of mass; MB.R.\#\#\#\# - MfN fossil reptile collection numbers; MfN - Museum für Naturkunde, Leibniz Institute for Research on Evolution and Biodiversity at the Humboldt University Berlin, Berlin, Germany; RBINS - Royal Belgian Institute of Sciences, Brussels, Belgium; SMA - Sauriermuseum Aathal, Aathal-Seegräben, Switzerland.

\section{Material and methods}

\subsection{Bone and skeletal mount 3-D scans}

The MfN's Dinosaur Hall displays a mounted skeleton of Kentrosaurus aethiopicus Hennig, 1915, which consists of the lectotype of $K$. aethiopicus (Hennig, 1925; Janensch, 1925; Mallison, 2011c) and other material. The skeletal elements were described in detail by Hennig (1925), whereas the process of bone selection and mounting was described in Janensch (1925). The mount was dismantled in 2005, the bones re-conserved and the skeleton remounted in a different, more lively pose in 2007 by Research Casting International. The composition of elements remained unchanged. Those elements of the mount that are real fossil bone and the plaster skull were scanned with a high-resolution Minolta laser scanner during the re-conversation effort. Previously, these scans were used for a digital skeletal mount and a study of the motion range of the limbs and vertebral column (Mallison, 2010), and a CAD life reconstruction based on the digital skeletal mount for an assessment of the defence behaviour (Mallison, 2011a), and the volumetric model is re-used here. Other research using the 3-D files is currently being published (Maidment et al., 2013, 2014).

The Stegosaurus armatus model is based on a mounted cast of a Stegosaurus in the RBINS. The skeleton is a composite made from casts of bones in the collection of the SMA, which houses several partial individuals. The mount was photographed with a Canon EOS 400D digital SLR camera, using a SIMGA $28-80 \mathrm{~mm}$ lens. Photographs were taken from all around the front, right side and back of the mount, but only a handful of photographs could be taken from the left side, due to the placement of the mount in the museum exhibition.

In total 80 photos were used for a photogrammetric reconstruction (see Falkingham, 2011, for a description of the method) in PhotoScan Professional by Agisoft, pre-release of Version 1.0.0. Alignment was performed using "high" accuracy and "disabled" pair selection, with a point limit of 10000 . The alignment of 76 photos (4 were not aligned) was visually inspected in-program for plausibility, and found to be acceptable. A dense point cloud was calculated using the settings quality "high" and depth filtering "moderate". Erroneous points and model parts not pertaining to the mounted skeleton were manually selected and deleted in PhotoScan. The left half of the skeleton was found to be badly modelled in parts (skull, ribcage), but of good quality in the tail. The right half and the plates had sufficient points for wrapping a useable surface. Therefore, the left side of the trunk was deleted. The point cloud was then meshed in Geomagic 10 to create a mesh with $\sim 1$ million polygons.

For creation of the digital 3-D model of the living animal, the mesh was then reduced to $25 \%$ polygon count ( $\sim 260.000$ polygons, using "fix boundaries" option), sectioned (i.e. limbs and osteoderms were made separate entities), imported into Rhinoceros 5.0, and straightened into a standing pose with vertical limbs and no lateral flexion of the vertebral column.

\subsection{Life reconstruction}

The CAD 3-D life reconstruction of Kentrosaurus used here is based on an unfinished version of that used in Mallison (2011a). The underlying skeletal data (high-resolution laser scans of the mounted skeleton in the $\mathrm{MfN}$ ) are figured in Mallison (2011c, Fig. 1). It was created in Rhinoceros NURBS Modelling for Windows 4.0 and 5.0 (McNeel \& Associates). For this study, slight changes were made to a version of the model that had not undergone sectioning into functional units.

Soft tissue extents were based on own dissection data, cross-section photographs of Alligator mississippiensis provided by R. Wilhite, CT scans and X-ray images of extant sauropsids, and data from Allen et al. (2009). The Supplement contains the files necessary to reconstruct the entire model.

The same model was used to create a model of Stegosaurus. Superposition of the 3-D model of the mounted cast and the finished life reconstruction model of Kentrosaurus showed that only modest modifications were required to fit the life model to the skeleton, namely a lengthening of the trunk section and the limbs (affected using the Scale1D command in Rhinoceros 5.0, which stretches a part along one axis only), as well as a ventral expansion of the trunk segment and tail base segment caused by the longer ischia of Stegosaurus. The latter point was not altered, resulting in an undersized trunk for the Stegosaurus model, 


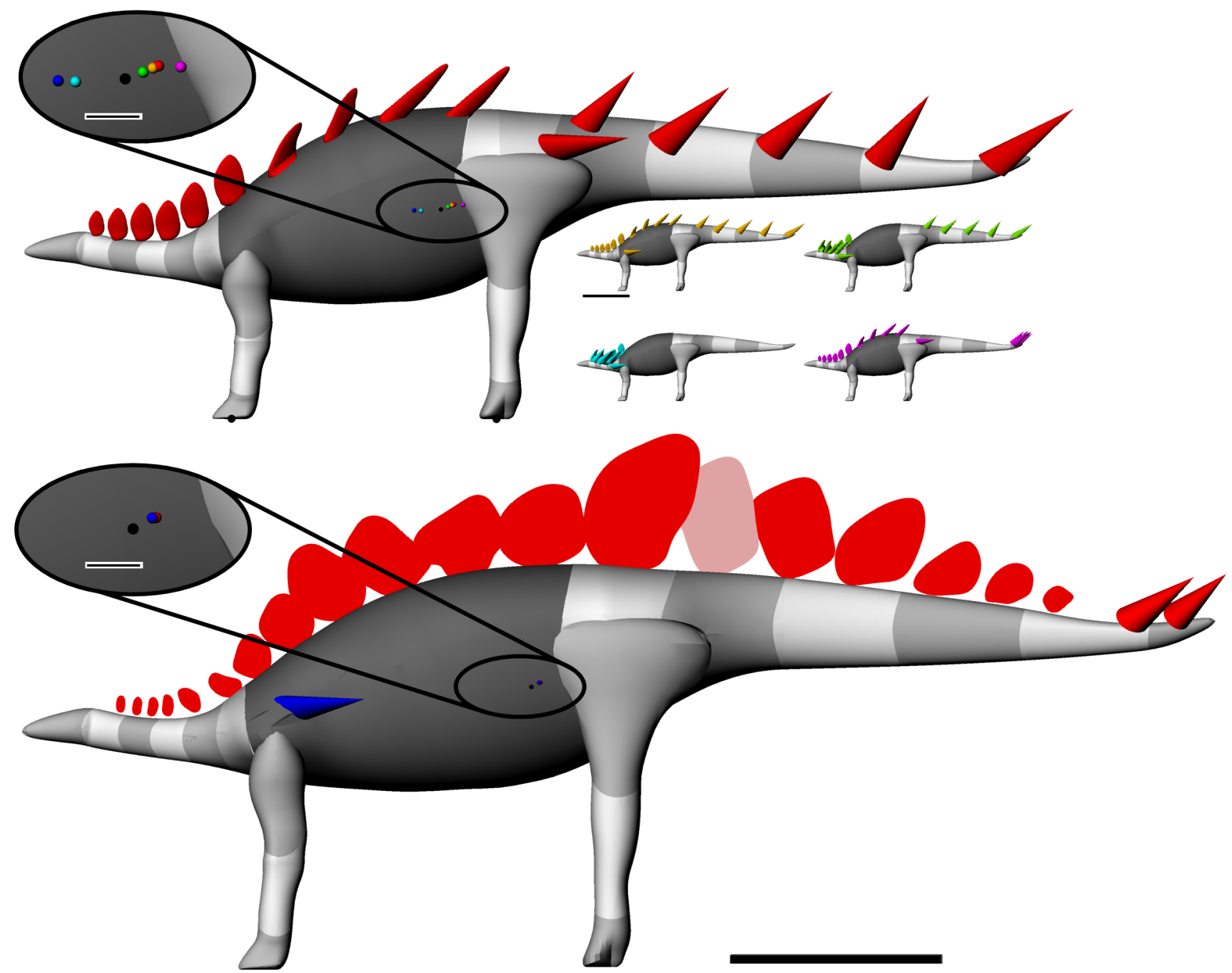

Figure 1. The CAD models of Kentrosaurus (top) and Stegosaurus (bottom). Kentrosaurus: main part depicts osteoderms (red) in distribution as reconstructed by Janensch (1925) ("starting configuration" model); scale bar $=1 \mathrm{~m}$. Smaller versions below the tail depict other arrangements with more anterior COM: "hip spike to shoulder" (gold), "all trunk to neck" (green), "all to neck" (light blue), and with more posterior COM: "all tail to tip" (lilac). Colours correspond to dots marking COM positions of all model variants; black dot corresponds to animal without osteoderms. Stegosaurus: red osteoderms are known from fossil "Sarah"; pink plate is reconstructed. Dots marking COM positions: black - without osteoderms, red - with osteoderms, dark blue - with osteoderms and additional shoulder spike. Enlarged inset scale bars for both models $=10 \mathrm{~cm}$.

favouring the hypotheses of Maidment et al. (2012) as a lighter trunk increases the effect of osteoderm weight on COM position.

The plates were based on a nearly complete subadult Stegosaurus armatus skeleton nicknamed "Sarah" (Siber and Möckli, 2009). Note that Siber and Möckli (2009) identify "Sarah" as S. stenops, although Maidment et al. (2008) consider $S$. stenops to be a junior synonym of $S$. armatus. The SMA holds a cast of this specimen (SMA R2218/01). Siber and Möckli (2009) figured the quarry map, which shows 18 plates and 4 spikes, most of them in situ. It is highly unlikely, based on the excavation record as described in Siber and
Möckli (2009), that more than one or at most two plates are missing. Hypothesis 1 of Maidment et al. (2012) is favoured by an underestimate of total osteoderm mass, as the relative impact of a scapular spike is larger if it makes up a larger percentage of overall weight. Therefore, I here assume that the full count of plates was 19, adding one plate to fill an apparent gap in "Sarah" (contra S. Maidment, cited in Siber and Möckli, 2009, who suggested an even number, and therefore 20 plates should have been present - although an even number is likely, adding another plate would weaken Hypothesis 1 in testing). 
Siber and Möckli (2009, p. 44) show all plates in one figure with scale. This image was used as a background image in Rhinoceros 5.0, scaled, and the plates were outlined with curves. These outlines were drawn generously, to take missing non-bony (keratinous) and bony parts into account. Then, two copies of each the curves were moved laterally to create thickness, with neck and distal tail plates given a thickness around $10 \mathrm{~mm}$, anterior trunk plates a thickness of $\sim 12$ to $15 \mathrm{~mm}$, and posterior trunk and anterior tail plates a thickness of 15 to $18 \mathrm{~mm}$. All these values are intentional underestimates, again favouring Hypothesis 1 . The resulting curve triplets were connected into a surface via the "loft" command. The resulting flat shapes were closed using the "cap" command. The plates were arranged along the dorsal margin of the body model (Fig. 1), but not tilted laterally, except for the tail spikes, because lateral displacements of the $\mathrm{COM}$ are not of interest here.

\subsection{Centre of mass determination}

The centre of mass was calculated assuming that stegosaurs did not have extensive air sacs as are seen in saurischian dinosaurs. To this day no pneumatic foramina are known in stegosaurs, and no other indicators for the presence of an avian-style lung (Butler and Barrett, 2012). If stegosaurs had a lung system with air sacs, they were likely not enlarged as in sauropods and theropods, and are thus modelled with sufficient accuracy if a generalized lung is taken into account. The lung was factored into the trunk segment by adjusting the density of the segment (see below).

The CAD model was sectioned into units that can be assumed to have uniform densities: limbs, tail, neck, skull, anterior trunk, and posterior trunk (hip). The osteoderms were all kept as separate entities as well.

Rhinoceros 5.0 can calculate the geometric centre of a 3D body or a group of bodies (VolumeCentroid command), so that the individual centre of mass for a body or set of bodies with equal density can be directly computed in the program. However, variations in the density of these objects are not taken into account. Because of the differences in density between the various body sections, for COM calculation all bodies were multiplied depending on their individual densities. Of those composed primarily of bone (osteoderms; density of $2 \mathrm{~kg} \mathrm{~L}^{-1}$; this overestimates osteoderm weight slightly as the average density of bone is $\sim 1.9 \mathrm{~kg} \mathrm{~L}^{-1}$; Currey, 2002), 10 copies each were used; those composed of bone and large amounts of muscle tissue (skull, tail, limbs, hip segment; density of $1.2 \mathrm{~kg} \mathrm{~L}^{-1}$; estimate based on ratio of volume of bones to volume of reconstructed soft tissues) 6 copies; of those composed of bone, muscle and less dense tissues such as the trachea and lung 4 copies (neck, trunk; density of $0.8 \mathrm{~kg} \mathrm{~L}^{-1}$; based on a lung volume percentage of the trunk of $\sim 9 \%$, calculated from values in Gunga et al., 2007). Because density and volume both affect the resulting mass, and because the volume can only be a rough estimate, a more de- tailed determination of densities would not add any reliability to the estimate.

By grouping all copies of all sections together, Rhinoceros 5.0 can directly calculate the centre of mass of the entire assembly as if the various parts were present only once each, but had different densities proportional to the ratio of copies used. Centre of mass position was calculated for the entire animal without any osteoderms, and for the osteoderm placement variations discussed below.

Rhinoceros 5.0 delivers the COM data in the form of $X$, $Y, Z$ coordinates and automatically adds a point object to the file. A small sphere was placed in the model at the appropriate position to better visualize COM position. Additionally, the percentage of body weight carried by the forelimbs and hindlimbs was calculated based on the horizontal distance between the COM and the points of support for the hindfeet and forefeet.

\subsection{Osteoderm placement variation}

Values for the different osteoderm distributions and the relative amount of body weight supported by each limb pair are given in Table 1, and Fig. 1 shows the visual plot of the COM in the differing models. The distributions for Kentrosaurus were chosen as follows:

- "starting configuration" - according to the best estimate by Hennig and Janensch, who collaborated on the creation of the MfN mount (Janensch, 1925).

- "hip spike to shoulder" - as above, but with the parasacral spike positioned on the shoulder. This position exemplifies the osteoderm placement difference suggested by Maidment et al. (2012) as a potential explanation of the humerus robustness differences between Kentrosaurus and Stegosaurus.

- "trunk to neck" - all osteoderms located on the neck. This is an unrealistic distribution that would require that Janensch (1925) was wrong about those osteoderms for which no field evidence of their location on the animal was found.

- "all to neck" - an unrealistic placement that attempts to create a significant COM displacement by assigning all osteoderms to the neck, including those for which evidence of a position on the tail was found in the field. Hennig (1925) specifically mentions four pairs of spikes found in close association with an articulated tail, and a tail tip with paired spikes in articulation is figured in Mallison (2011a, Fig. 3). - "all tail to tip" - retains the starting configuration except for all non-tail tip spikes on the tail, which were moved to the tail tip.

For Stegosaurus, "starting configuration" corresponds to a relatively even spread of osteoderms along the dorsal margin of the animal, based on the quarry map published by Siber and Möckli (2009). An additional configuration ("with de novo shoulder spikes") added the two parasacral spikes of Kentrosaurus to Stegosaurus, as parascapular spikes. 
Table 1. COM shifts for osteoderm placement variants.

\begin{tabular}{lrrrr}
\hline Model version & $\begin{array}{r}\text { Distance COM } \\
\text { to COM without } \\
\text { spikes (mm) }\end{array}$ & $\begin{array}{r}\text { Distance to } \\
\text { hindlimb support } \\
\text { point (mm) }\end{array}$ & $\begin{array}{r}\% \text { of } \\
\text { weight on } \\
\text { forelimbs }\end{array}$ & $\begin{array}{r}\text { \% of } \\
\text { weight on } \\
\text { hindlimbs }\end{array}$ \\
\hline Kentrosaurus & & & & \\
\hline No spike & 0 & 263 & 21 & 79 \\
Starting configuration & -63 & 200 & 16 & 84 \\
Hip spike to shoulder & -53 & 210 & 17 & 83 \\
Trunk to neck & -34 & 229 & 18 & 82 \\
All to neck & 94 & 357 & 28 & 72 \\
All tail to tip & 106 & 157 & 13 & 87 \\
\hline Stegosaurus & & & & \\
\hline No spike & 0 & 337 & 21 & 79 \\
Starting configuration & -37 & 293 & 18 & 82 \\
With de novo shoulder spike & -43 & 300 & 19 & 81 \\
\hline
\end{tabular}

\section{Results}

As can be seen from Fig. 1 and Table 1, the COM position in both modelled taxa is nearly identical, with the hindlimbs in both models supporting $\sim 79 \%$ of the total body weight without osteoderms, and $82 \%$ (Kentrosaurus) and $84 \%$ (Stegosaurus) with the likely osteoderm distribution. The posterior COM location found here in stegosaurs is in agreement with a previous study by Henderson (1999). Scapular spikes in both taxa shifts $\sim 1 \%$ of body weight from the hindlimbs to the forelimbs.

Moving the pair of parasacral spikes from the hip to the pectoral area in Kentrosaurus results in a cranial shift of the COM by $10 \mathrm{~mm}$, relative to a "wheel base" (distance between fore- and hindfeet) of $1255 \mathrm{~mm}$, moving $+1 \%$ of the body weight to the forefeet. In Stegosaurus, adding scapular spikes ex novo also shifts $\sim 1 \%$ of body weight from the hindfeet to the forefeet. Shifting all osteoderms of Kentrosaurus with unknown provenance to the neck - an unrealistic but theoretically possible scenario - shifts the COM cranially by $29 \mathrm{~mm}$ compared to the reconstruction Janensch (1925) deemed likely. The latter version places an additional $2 \%$ of the body weight on the front limbs. A significant shift, here deemed to exceed the equivalent to adding $>10 \%$ of body weight to the forelimbs, requires moving all osteoderms to the neck, including those for which the position along the tail is known from articulated finds.

Thus, the explanation proposed by Maidment et al. (2012) for the observed difference in robustness of the humeri of Kentrosaurus and Stegosaurus, Hypothesis 1, is falsified.

With regards to Hypothesis 2, it becomes apparent that the lever arm of the body for a rotation around the acetabulum into an upright pose is small, here estimated as $\sim 20 \mathrm{~cm}$ in Kentrosaurus and $\sim 29 \mathrm{~cm}$ in Stegosaurus for the likely osteoderm distribution models.

\section{Discussion}

\subsection{More robust humeri in smaller stegosaurs}

Obviously, an assessment of how shifting osteoderms influences COM position can be easily performed using simple mathematical equations, and does not require 3-D modelling. Given the small percentage of total body weight the spikes and plates make up, it is immediately obvious that - given our overall quite good knowledge of their distribution in stegosaurs from (semi-)articulated finds like USNM 4934 (Gilmore, 1914) and DMNH 2818 (Carpenter, 1998) - their various distributions did not have a major influence on the proportion of the weight supported by the forelimbs. However, the 3-D models illustrate this point very well, and their creation based on already existing scans and models was hardly more time-consuming than an investigation via mathematical formulas would have been.

Overall, the COM shift from even significant redistribution of osteoderms is minimal. Their total volume is here estimated as roughly $0.038 \mathrm{~m}^{3}$, compared to a volume of slightly over $1.039 \mathrm{~m}^{3}$ for the animal without osteoderms. Total volume for the entire living animal is thus estimated as $\sim 1.077 \mathrm{~m}^{3}$, of which the osteoderms make up $\sim 3.5 \%$. Assuming that their density is twice that of the average density of the animal means they weigh around $7 \%$ of the total. Articulated finds of partial and complete stegosaurs show that, overall, osteoderms were distributed so that the entire animal's dorsal outline was more or less regularly covered by them. Therefore, variation between genera and species can only have affected a small part of the $7 \%$, and thus not have influenced the COM position. This is further confirmed by the ridiculous distribution versions required to create a significant COM shift (Fig. 1), which do not resemble any known fossil, and are also physically impossible due to 
insufficient space on the animal's surface for the anteriorly bunched osteoderms.

One question that must be discussed in this context is the sensitivity of the analysis presented here or any similar approach to the inherent inaccuracies of body mass estimation. Obviously, as the trunk, thighs and proximal tail are by far the largest volumes in the body, small variations in soft tissue estimation can have a significant influence on the total mass of the animal. However, even if we arbitrarily assume that the total body volume was half of what was reconstructed here (i.e. if the soft tissues on the body were massively overestimated), the COM motions induced by moving osteoderms in realistic versions would still remain minimal (i.e. below $4 \%$ of additional weight supported by the forelimbs).

The uncertainties involved in the soft tissue reconstructions performed here are massive, but as both models were shaped based on highly similar starting data (3-D skeletons) and using similar sets of assumptions, it is likely that the two models at least are imperfect in similar ways. Relative differences between them therefore should be more reliable than the exact reconstructed volumes individually. Both models recover the COM with and without osteoderms in highly similar positions (Table 1), despite the differences in proportions especially of the trunk between the two taxa. Two taxa, one large and one small, are obviously insufficient to test the suggestion of Maidment et al. (2012) that the more robust humeri of small stegosaur taxa "could be due to changes in the center of mass" generally (i.e. by changes caused by other factors than osteoderm placement). However, the presented data and a previous COM estimate (Henderson, 1999) agree, and unless the unusually long-necked stegosaur Miragaia (Mateus et al., 2009) was highly unusually built in the unknown posterior half, it seems unlikely that other taxa differed much from those tested here in overall body shape and mass distribution. Therefore, we must look for alternative explanations for the apparent inverse scaling of forelimb strength with body size described by Maidment et al. (2012). Defence behaviour involving rapid lateral acceleration of the anterior body has been suggested (Bakker, 1986), and remains a candidate, although it may not explain the observed correlation with size.

\subsection{Generally robust humeri in stegosaurs}

The COM of stegosaurs was very likely not located more anteriorly than in other ornithischians, as models of Iguanodon, Triceratops and Stegosaurus by Henderson (1999) show. The generally same COM position was here found for Stegosaurus and Kentrosaurus. Therefore, the hypothesis of Maidment et al. (2012) that "the center of mass of stegosaurs was located more anteriorly than in other ornithischians" is not supported, and cannot explain the robustness of stegosaur humeri, as Maidment et al. (2012) already pointed out.

Maidment et al. (2012) suggest instead that increased stress on the humerus was generated during rearing as a result of pushing off from the ground (Hypothesis 2). Malli- son (2011a) estimated the mass and COM position of Kentrosaurus, finding that the COM may have been located so far posteriorly that for reasonable model variants 80 to $85 \%$ of the body weight was supported by the hindlimbs, identical to the results found here for both Kentrosaurus and Stegosaurus. Previous research on the COM position in Stegosaurus has found a similar weight distribution (Henderson, 1999). Mallison (2010) also found a tripodal pose to be plausible for Kentrosaurus from a skeletal range of motion aspect. However, given the very posterior position of the COM it seems highly unlikely that high forces in the front limbs were necessary to adopt a tripodal pose, a situation similar to that in diplodocid sauropods (Mallison, 2011b). Even though the total mass of a living stegosaur was significant (Mallison (2011a) estimated a volume of $\sim 1070 \mathrm{~L}$ for Kentrosaurus; for Stegosaurus Henderson (1999) estimated a mass of $2811 \mathrm{~kg}$; Alexander (1989) $3.1 \mathrm{t}$; Colbert (1962) $1.78 \mathrm{t}$ at a density of $0.9 \mathrm{~kg} \mathrm{~L}^{-1}$ ), if such a mass were accelerated to rotate around a point via a short lever arm, the forces required to do so would be small when applied via a large moment arm. In the Kentrosaurus model, the forelimb moment arm is nearly $1 \mathrm{~m}$ longer than the average COM moment arm for a rotation around the acetabulum, or roughly five times as large. At a 5:1 advantage, even a modest push would result in sufficient rotation to lift an animal into a tripodal stance, especially considering the significant strength of the caudofemoral musculature (evident from the distal placement of the fourth trochanter of the femur roughly at mid-shaft, as is common in most quadrupedal ornithischians, but not in theropod dinosaurs for example), which on static limbs would also rotate the body into an upright position. Forces encountered during locomotion may well have required forelimb strengths sufficient to create such an upward push by combining both limbs' actions. Therefore, an upright stance for feeding or fighting is unlikely to have been the cause of proportionally unusually strong forelimbs in stegosaurs compared to other ornithischians. Again, defence behaviour may be a more powerful explanation, as it may involve rapid accelerations co-directional or counterdirectional to the tail, which may have been created and countered by the forelimbs.

\section{Supplementary material related to this article is available online at http://www.foss-rec.net/17/33/2014/ fr-17-33-2014-supplement.zip.}

Acknowledgements. The author thanks D. Schwarz-Wings (MfN) for access to specimens and relentless cataloguing of stegosaur material in the MfN collection. An anonymous reviewer and S. C. R. Maidment made many helpful comments on a previous draft, which have improved the final manuscript significantly. I am especially grateful to S. C. R. Maidment for her extremely 
professional conduct, which is a rare and shining example of how researchers should behave when their hypotheses are in question. She also caught several mistakes and inaccuracies in the second draft, and I am thankful for her patience and diligence. S. Hayashi is thanked for providing photographs of Chinese stegosaur specimens.

Edited by: F. Witzmann

Reviewed by: S. Maidment and one anonymous referee

\section{References}

Alexander, R. M.: Dynamics of dinosaurs and other extinct giants, Columbia University Press, New York, 1989.

Allen, V. R., Paxton, H. and Hutchinson, J. R.: Variation of center of mass estimates for extant sauropsids and its importance for reconstructing inertial properties of extinct archosaurs, Anat. Rec., 292, 1442-1461, 2009.

Bakker, R. T.: The Dinosaur Heresis, William Morrow and Co., New York, 1986.

Butler, R. J. and Barrett, P. M.: Reassessment of the evidence for postcranial skeletal pneumaticity in Triassic archosaurs, and the early evolution of the avian respiratory system, PLOS ONE, 7, e34094, doi:10.1371/journal.pone.0034094, 2012.

Carpenter, K.: Armor of Stegosaurus stenops, and the taphonomic history of a new specimen from Garden Park Colorado, Up. Jurass. Morrison Form. Interdiscip. Study Part 1 Mod. Geol., 22, 127-144, 1998.

Colbert, E. H.: The weights of dinosaurs, Am. Mus. Novit., 2076, 1-17, 1962.

Currey, J. D.: Bones: Structure and Mechanics, Princeton University Press, New Jersey, 2002.

Falkingham, P. L.: Acquisition of high resolution three-dimensional models using free, open-source, photogrammetric software, Palaeontol. Electron., 15.1.1T, 2011.

Galton, P. M.: The postcranial anatomy of stegosaurian dinosaur Kentrosaurus from the Upper Jurassic of Tanzania, East Africa, Geol. Palaeontol., 15, 139-165, 1982.

Galton, P. M. and Upchurch, P.: Stegosauria, in: The Dinosauria, 2nd Edition, edited by: Weishampel, D. B., Dodson, P., and Osmolska, H., University of California Press, Berkeley, 343-362, 2004.

Gilmore, C. W.: Osteology of the armored Dinosauria in the United States National Museum, with special reference to the genus Stegosaurus, U.S. Natl. Mus. Bull., 89, 1-136, 1914.

Gunga, H.-C., Suthau, T., Bellmann, A., Friedrich, A., Schwanebeck, T., Stoinski, S., Trippel, T., Kirsch, K. and Hellwich, O.: Body mass estimations for Plateosaurus engelhardti using laser scanning and 3D reconstruction methods, Naturwissenschaften, 94, 623-630, 2007.

Henderson, D. M.: Estimating the masses and centers of mass of extinct animals by 3D mathematical slicing, Paleobiology, 25, 88-106, 1999.
Hennig, E.: Kentrosaurus aethiopicus der Stegosauride des Tendaguru, Sitzungsberichte Ges. Naturforschender Freunde Zu Berl., 1915, 219-247, 1915.

Hennig, E.: Kentrurosaurus aethiopicus Die Stegosaurier-Funde vom Tendaguru, Deutsch-Ostafrika, Palaeontographica, Supplement 7, 101-254, 1925.

Janensch, W.: Ein aufgestelltes Skelett des Stegosauriers Kentrurosaurus aethiopicus HENNIG 1915 aus den TendaguruSchichten Deutsch-Ostafrikas, Palaeontographica, Supplement 7, 255-276, 1925.

Maidment, S. C. R., Norman, D. B., Barret, P. M., and Upchurch, P.: Systematics and phylogeny of Stegosauria (Dinosauria: Ornithischia), J. Syst. Palaeontol., 6, 367-407, doi:10.1017/S1477201908002459, 2008.

Maidment, S. C. R., Linton, D. H., Upchurch, P., and Barrett, P. M.: Limb-bone scaling indicates diverse stance and gait in quadrupedal ornithischian dinosaurs, PLOS ONE, 7, e36904, doi:10.1371/journal.pone.0036904, 2012.

Maidment, S. C. R., Bates, K. T., and Barrett, P. M.: Threedimensional computational modeling of locomotor muscle moment arms in Edmontosaurus (Dinosauria: Hadrosauridae) and comparisons with other archosaurs, in: Hadrosaurs, edited by: Evans, D. E. and Eberth, D. C., Indiana University Press, in press, 2014.

Maidment, S. C. R., Bates, K. T., Falkingham, P. L., VanBuren, C., Arbour, V. M., and Barrett, P. M.: Locomotion in ornithischian dinosaurs: an assessment using three-dimensional computational modeling, Biol. Rev., doi:10.1111/brv.12071, 2013b.

Mallison, H.: CAD assessment of the posture and range of motion of Kentrosaurus aethiopicus Hennig 1915, Swiss J. Geosci., 103, 211-233, doi:10.1007/s00015-010-0024-2, 2010.

Mallison, H.: Defense capabilities of Kentrosaurus aethiopicus Hennig, 1915, Palaeontol. Electron., 14, 10A, 2011a.

Mallison, H.: Rearing Giants - kinetic/dynamic modeling of sauropod bipedal and tripodal poses, in: Biology of the Sauropod Dinosaurs: Understanding the life of giants, edited by: Klein, N., Remes, K., Gee, C. T., and Sander, P. M., Indiana University Press, Bloomington, 237-250, 2011b.

Mallison, H.: The real lectotype of Kentrosaurus aethiopicus Hennig 1915, Neues Jahrb. für Geol. Paläontol. Abh., 259, 197-206, 2011c.

Mateus, O., Maidment, S. C. R., and Christiansen, N. A.: A new long-necked "sauropod-mimic" stegosaur and the evolution of the plated dinosaurs, Proc. R. Soc. B Biol. Sci., 276, 1815-1821, doi:10.1098/rspb.2008.1909, 2009.

Ouyang, H.: [Discovery of Gigantspinosaurus sichuanensis and its scapular spine orientation.], Abstr. Summ. Youth Acad. Symp. New Discov. Ideas Stratigr. Paleontol. Nanjing Dec 1992, 47-49, 1992.

Siber, H. J. and Möckli, U.: The Stegosaurs of the Sauriermuseum Aathal, Sauriermuseum Aathal, Aathal, Switzerland, 2009. 